\section{Changes in EMS Injury Rates and Patterns Caused by Patient Treatment in a Moving Vehicle}

\author{
O'Connor $R^{*+}$ Megargel $R$, *+** Thomas $W^{* *}$ \\ * Department of Emergency Medicine, \\ The Medical Center of Delaware, Wilmington, Delaware, USA \\ ** EMS Division, Department of Public Safety, \\ New Castle County, New Castle, Delaware, USA \\ + Office of Paramedic Administration, \\ Division of Public Health, Dover, Delaware, USA
}

Purpose: Prehospital management of critically ill patients by EMS providers often requires expedient transport. Certain treatments, once performed at the scene, now are deferred or performed in a moving vehicle. This study defines whether treatment conducted in a moving vehicle increases the risk of injury to EMS providers.

Methods: Paramedics within this system are required to report all on-the-job injuries, and identify contributing factors, such as vehicle movement or faulty equipment. In June 1991, a new trauma protocol was instituted which specified rapid transport and treatment while en route. The reported injury rates from a one year period before and after initiation of this trauma protocol was examined to determine the effect of vehicle movement on EMS injuries.

Results: During the period prior to initiation of the new protocol, 12 injuries were reported. None were caused by vehicle movement. Four injuries involved needle sticks. After initiation of the new protocol, 14 of 29 reported injuries were due to vehicle movement. Of the 15 needle sticks in this group, 11 were due to vehicle movement. Both the increased number of injuries $\left(\mathrm{x}^{2}=6.78, p<.01\right)$ and the increase in needle sticks $\left(\mathrm{x}^{2}=4.2, p<.05\right)$ caused by vehicle movement were significant. The $99 \%$ confidence intervals for differences between the proportion of injuries caused by vehicle movement were $(0.25$, $0.71)$ for total injuries and $(0.17,1.00)$ for needle sticks.

Conclusions: Therapy performed by EMS providers in a moving ambulance should be kept to an absolute minimum. EMS personnel should not use exposed needles or move about unless the vehicle is stopped.

\section{Standing Orders-Does This System Decrease the Prehospital Patient Care Error Rate?}

\author{
Holliman J, Wuerz RC, Meador SA \\ University Hospital, The Milton S. Hershey Medical Center \\ The Pennsylvania State University \\ Hershey, Pennsylvania, USA
}

Objective: The aim of this study was to compare the error rates of physician medical commanders and paramedics before and after implementation of a standing orders protocol system for patient care by paramedics.

Design: Physician review of prehospital trip sheets conducted prospectively as part of an ongoing quality assurance $(Q / A)$ program.

Setting: An urban paramedic service in the northeastern United States

Participants: 2,001 advanced life support run reports from 1 April 1991, the start date of the protocol system, through 31 January 1992 were reviewed as part of the $Q / A$ program.

Interventions: Errors in patient care (failure to administer an indicated treatment or medication or performing inappropriate or excessive treatment) by medical command physicians and by paramedics were recorded. The errors were compared to the medical command errors determined from a previous study encompassing transports from September 1988 through December 1990, at which time paramedics were required to obtain medical command for most treatments.

Results: Medical command errors decreased from $4.4 \%$ to $1.2 \%$ of runs after the standing orders system was adopted. Paramedic error rates remained at $<0.5 \%$ in both systems. Mean paramedic on-scene time interval decreased by 68 seconds with use of the standing orders system.

Conclusions: Use of standing orders to direct initial patient care by paramedics resulted in significant decrease in the treatment error rate by medical command physicians, no change in the low paramedic error rate, and slightly decreased on-scene times. Use of standing orders may improve efficiency of prehospital care without compromising quality of patient care. 